Camille Cornand, Frank Heinemann

\title{
Limited higher order beliefs and the welfare effects of public information
}

Journal article | Accepted manuscript (Postprint)

This version is available at https://doi.org/10.14279/depositonce-9229

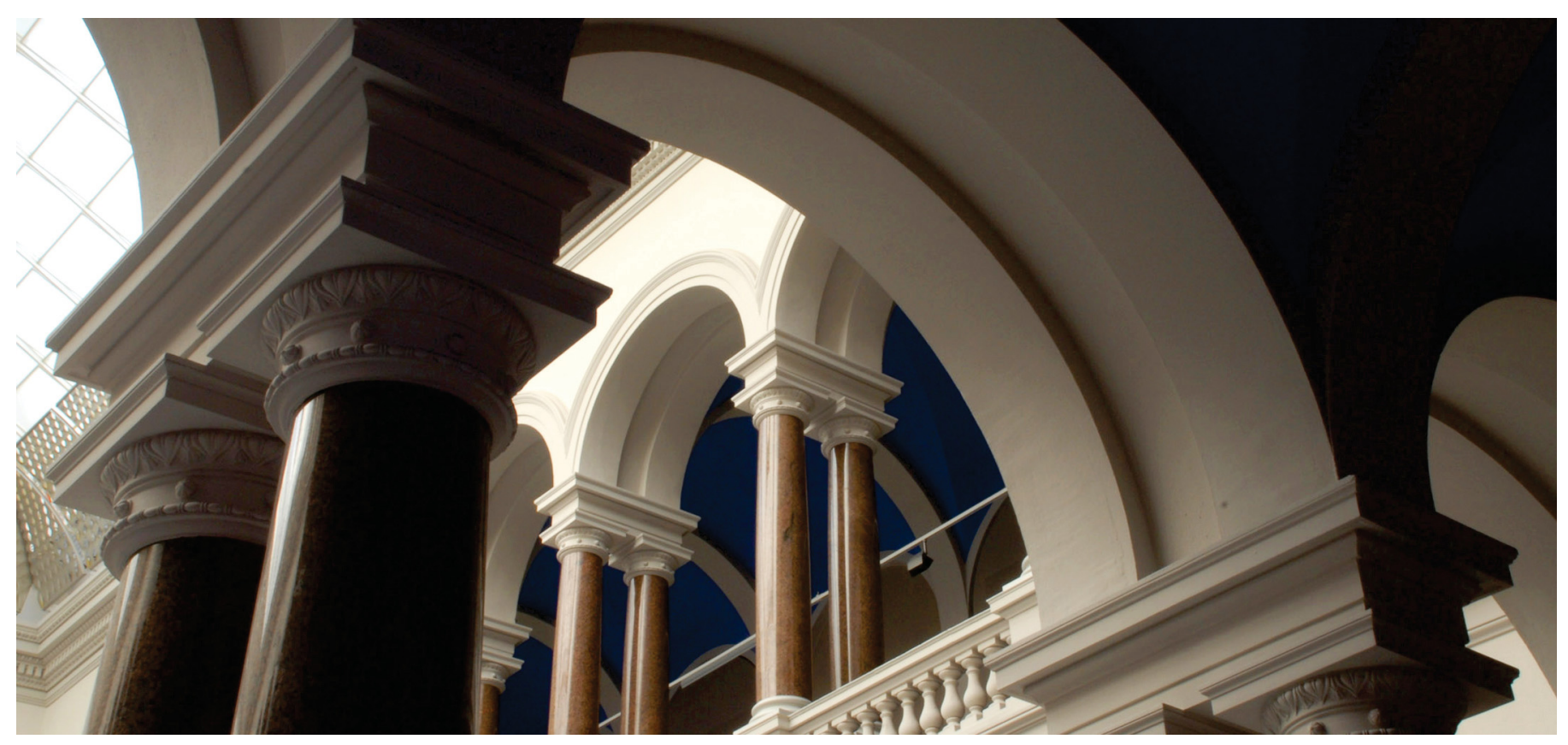

Cornand, C., \& Heinemann, F. (2015). Limited higher order beliefs and the welfare effects of public

information. Journal of Economic Studies, 42(6), 1005-1028. https://doi.org/10.1108/jes-08-2015-0142 


\title{
Limited higher order beliefs and the welfare effects of public information*
}

\author{
Camille Cornand and Frank Heinemann
}

August 6, 2015

\begin{abstract}
In games with strategic complementarities, public information about the state of the world has a larger impact on equilibrium actions than private information of the same precision, because the former is more informative about the likely behavior of others. This may lead to welfare-reducing 'overreactions' to public signals as shown by Morris and Shin (2002). Recent experiments on games with strategic complementarities show that subjects attach a lower weight to public signals than theoretically predicted. Aggregate behavior can be better explained by a cognitive hierarchy model where subjects employ limited levels of reasoning. This paper analyzes the welfare effects of public information under limited levels of reasoning and argues that for strategies according with experimental evidence, public information that is more precise than private information cannot reduce welfare, unless the policy maker has instruments that are perfect substitutes to private actions.
\end{abstract}

JEL Classification: D82, D83, E52, E58.

Keywords: coordination games, strategic uncertainty, private information, public information, higherorder beliefs, levels of reasoning.

\footnotetext{
* We are thankful to the ANR-DFG joint grant for financial support (ANR-12-FRAL-0013-01). This research was performed within the framework of the LABEX CORTEX (ANR-11-LABX-0042) of Universite de Lyon, within the program "Investissements d'Avenir" (ANR-11-IDEX-007) operated by the French National Research Agency (ANR).

Camille Cornand - Université de Lyon, Lyon, F-69007, France ; CNRS, GATE Lyon Saint-Etienne, Ecully, F69130, France; email: cornand@gate.cnrs.fr.

Frank Heinemann - Technische Universität Berlin, Chair of Macroeconomics, H 52 - Straße des 17. Juni 135 10623 Berlin, Germany; Email: frank.heinemann@tu-berlin.de.
} 


\section{1 - Introduction}

There has been an extensive debate about the welfare effects of transparency in environments with strategic complementarities, started by the seminal paper by Morris and Shin (2002). Strategic complementarities are widespread in macroeconomics, models of financial crises, price competition, investment in network goods, and many other economic situations. In games with strategic complementarities, public and private information about an uncertain state of the world have distinct effects, because information that is shared by several agents is more informative about the likely beliefs and actions of others than private information. Given that public signals are more informative, rational players put a higher weight on these signals than on private signals of equal precision. In equilibrium, the weight on public signals is even higher, because rational players account for the rationality of others and the attached weights are strategic complements.

This may lead markets to 'overreact' to public signals and raise price volatility. In this context, institutions such as central banks committing to provide noisy public information may reduce expected welfare. The aim of this paper is to reconsider the extent to which public information may be detrimental to welfare when accounting for the fact that 'real' agents are not fully rational. Recent experiments on games with strategic complementarities show that subjects attach a lower weight to public signals than theoretically predicted. Aggregate behavior can be better explained by a cognitive hierarchy model where subjects employ limited levels of reasoning. This paper analyzes the welfare effects of public information under such limited levels of reasoning and argues that for strategies according with experimental evidence, public information that is more precise than private information cannot reduce welfare, unless the policy maker has instruments that are perfect substitutes to private actions.

Morris and Shin (2002) present a stylized beauty-contest game with weak strategic complementarities for analyzing the welfare effects of public and private information. In their game, an agent's action is a weighted average of a public and a private signal. The equilibrium weight attached to the public signal is higher than its relative precision. In their welfare analysis, Morris and Shin concentrate on a case where those who predict the average opinion better gain at the expense of those whose prediction is worse. Here, coordination is a zero-sum game. Morris and Shin show that raising the precision of public signals may reduce expected welfare while raising the precision of private signals is always welfare improving.

Cornand and Heinemann (2014) test the theoretical predictions of the model by Morris and Shin (2002) in a laboratory experiment. They measure the actual weights that subjects attach to public and private signals and find that observed weights are distributed around the predictions from a cognitive hierarchy model, where players take into account that other players receive the same public signals, but neglect that other players also account for others receiving the same public signals. Such a neglect of higher-order rationality has also been observed in many other experiments on beauty- 
contest games, cascade games, and coordination games with strategic complementarities. ${ }^{1}$ It seems to be a fairly robust result that actual players' choices in these games are distributed around the level-2 choice. In the definition that we apply, level-2 players respond in a rational way to the non-strategic part of a game and they account for other players responding rationally, but they neglect that other players also account for others' rationality. ${ }^{2}$

In this paper, we apply limited levels of reasoning to four variants of the model by Morris and Shin (2002) that represent prototypes, in the sense that most of the literature follows one of these model types. The main distinction between these models is whether coordination is socially desirable or not. We reconsider the welfare effects of public and private information in these models accounting for limited levels of reasoning as observed in recent experiments. ${ }^{3}$

Our main result shows that in the original model by Morris and Shin (2002), in which coordination is not socially desirable, public information is always welfare improving if strategies are derived from level-2 reasoning. The result also holds for weights associated with Level 1 or any mixture of levels up to Level 2. If, however, agents employ higher levels of reasoning, negative welfare effects of public information are possible. Since individual strategies are widely dispersed including some subjects with higher levels of reasoning - we check that for the mixture of strategies observed in Cornand and Heinemann (2014) a higher precision of public information increases welfare. Higher-order beliefs play a key role in explaining why the precision of public information might be bad. The higher the levels of reasoning, the higher is the weight that agents put on the public signal, and the more likely are welfare detrimental effects. For level-2 reasoning, agents account for the public signal providing more information about others' actions than private signals, but neglect that others are also putting higher weights on public signals. The welfare detrimental effects stemming from strategic behavior only kick in, when agents account for the higher weights of others, which requires more than 2 levels of reasoning.

Angeletos and Pavan (2007a, b) emphasize that the welfare effects of transparency are sensitive to the extent to which coordination is socially valuable. Hellwig (2005), Roca (2010), Lorenzoni (2010) and Woodford (2005) embed the mechanism of Morris and Shin (2002) into a

\footnotetext{
${ }^{1}$ Examples are Nagel (1995), Kübler and Weizsäcker (2004), and Baeriswyl and Cornand (2014, 2015). Shapiro, Shi, and Zillante (2014) argue that the predictive power of level- $k$ reasoning is positively related to the strength of the coordination motive and to the symmetry of information.

${ }^{2}$ Baeriswyl and Cornand (2015) detect that subjects have biased first-order beliefs, when receiving signals of different precision. They test the levels of reasoning starting with Level 1 as the elicited first-order belief.

${ }^{3}$ It seems intriguing to test the welfare effects of limited levels of reasoning directly. However, the utility function as stated by Morris and Shin (2002) contains three intertwined effects: a fundamental motive, a coordination motive and a competition motive. Cornand and Heinemann (2014), but also Baeriswyl and Cornand (2014, 2015) considered this triple task as too demanding for participants in the experiment and used a simpler game getting rid of the competition motive without affecting the equilibrium action. See Baeriswyl and Cornand (2014) for a more detailed discussion. In addition, a direct test would require different experiments for each of the four prototypes of the model that we can analyze using the results from just one experiment.
} 
macro-framework, where coordination is socially valuable. Here both, public and private information contribute to social welfare. The positive welfare effects of public signals in this model are robust to limited levels of reasoning. However, contrary to what the equilibrium theory predicts, increasing the precision of private signals may reduce expected welfare. The reason is that agents put a larger weight on private signals than is socially optimal. Higher precision of private signals raises the weight on private signals, which impedes desirable coordination.

Cornand and Heinemann (2008) introduce a concept of partial publicity by allowing for information that is provided to just a fraction of agents. They show that optimal policy requires to provide public signals with highest possible precision, but eventually not to all agents. Restricting signals to be partially public leads to a higher welfare than providing public signals to all agents with restricted precision. These qualitative results are robust to limited levels of reasoning, However, we find that the optimal degree of publicity is higher if agents apply level-2 reasoning than for equilibrium strategies. If public information is more precise than private information, then full transparency is optimal.

Finally, we reconsider a model by James and Lawler (2011) in which the government can take policy actions against some fundamental shock. Since policy and private actions are perfect substitutes with respect to neutralizing aggregate shocks, the government can provide the optimal response to its own information. Private responses are still required to account for the additional information contained in agents' private signals. This distribution of tasks achieves the first best. If the government discloses its own information as a public signal to private agents, agents reduce the weight they put on the private signal and, thus, private information enters the total response of the economy with a weight that is suboptimally small. For this reason, it is always optimal to withhold public information completely. This argument only requires that subjects put weights on signals that differ from the social optimum. Thus, complete intransparency is also optimal if agents follow limited levels of reasoning (with levels above 1) or if they aggregate private and public signals with some bias as in Baeriswyl and Cornand (2015). For level-1 reasoning, however, transparency has no welfare effect, as it only shifts the adjustment from public to private actions without affecting the aggregate.

Overall, these results indicate that under limited levels of reasoning, (i) public information is less detrimental (if at all) to welfare than predicted by equilibrium theory, (ii) private information can be welfare reducing which is opposed to equilibrium theory.

In the remainder of this paper, we first present the original set-up by Morris and Shin (2002). Section 3 introduces limited levels of reasoning in this model and shows that the main result of Morris and Shin is not robust to level-2 reasoning. Sections $4-6$ analyze limited levels of reasoning when coordination is socially desirable (Section 4), information can be partially public (Section 5), and policy actions can substitute private actions (Section 6). Section 7 concludes. 


\section{2 - Welfare analysis in the Morris-Shin-model}

In the model of Morris and Shin (2002), there is a continuum of agents $i$. The payoff function for agent $i$ is given by

$$
u_{i}(a, \theta) \equiv-(1-r)\left(a_{i}-\theta\right)^{2}-r\left(L_{i}-\bar{L}\right),
$$

where $L_{i} \equiv \int_{0}^{1}\left(a_{j}-a_{i}\right)^{2} d j$ and $\bar{L} \equiv \int_{0}^{1} L_{j} d j$. The utility function for individual $i$ has two components: the first component is a standard quadratic loss in the distance between the underlying state $\theta$ and her/his action $a_{i}$. The second component is reminiscent of the Keynesian beauty contest; $0 \leq r<1$ is the weight on the beauty contest term. The loss is increasing in the distance between $i$ 's action and the average action of the whole population. Agents gain from predicting the average opinion better than others, but the beauty-contest is a zero-sum game. Social welfare, as defined by the (normalized) average of individual utilities, is given by

$$
W(a, \theta) \equiv \frac{1}{1-r} \int_{0}^{1} u_{i}(a, \theta) d i=-\int_{0}^{1}\left(a_{i}-\theta\right)^{2} d i .
$$

As a consequence, a social planner, who cares only about social welfare, seeks to keep all agents' actions close to state $\theta$. While coordination affects individual payoffs, it does not affect social welfare.

Agents face uncertainty concerning the state: $\theta$ has a uniform distribution on the reals and agents receive two kinds of signals that deviate from $\theta$ by independent error terms with normal distribution. Each agent receives a private signal $x_{i}=\theta+\varepsilon_{i}$ with precision $\beta$. Signals of distinct individuals are independent and the distribution of private signals is treated as exogenously given. Agents have access to a public signal $y=\theta+\eta$ with precision $\alpha$.

The public signal is given to all agents. While a social planner would prescribe that agents choose actions in accordance with the expected state of the world, optimizing agents put a higher weight on the public signal, because it is more helpful in predicting the actions of other agents than uncorrelated private signals. In equilibrium, prior expected welfare is given by

$$
E(W)=-\frac{\alpha+\beta(1-r)^{2}}{(\alpha+\beta(1-r))^{2}},
$$

which is decreasing in the precision of public signals $\alpha$, if $\alpha / \beta<(1-r)(2 r-1)$. Since agents attach a weight to the public signal that exceeds its relative precision, noisy public signals drag the mean of private actions away from the fundamental. This bias towards the public signal reduces welfare and may dominate the positive welfare effect of actions being based on more precise information. Increasing the precision of private information, however, is always beneficial. 
Svensson (2006) criticized the negative welfare effects of transparency as being unrealistic. If public information is withheld, $\alpha=0$, expected welfare is $-1 / \beta$. Using the restriction $r \in[0,1)$, Svensson shows that private information needs to be more precise than public information for expected welfare being smaller than $-1 / \beta$. Since empirical estimates like Romer and Romer (2000) found public forecasts to be better than private forecasts, the disclosure of these forecasts would always raise expected welfare. ${ }^{4}$

\section{3 - A cognitive hierarchy model on the use of public and private information}

Cornand and Heinemann (2014) present an experiment, in which subjects repeatedly play a beauty-contest game based on Morris and Shin (2002). In each round, each subject is randomly matched with another subject, and the payoff function is such that players want to choose actions that are close to an unknown fundamental but also close to each other. Each subject receives two signals on the fundamental, a private signal and a common signal. The common signal is the same for both subjects, and subjects know, which of the two signals is common. All signals have the same precision. Appendix A provides more details about the design of this experiment. Varying the relative importance of the coordination motive, Cornand and Heinemann measure the relative weights that subjects put on the two signals. ${ }^{5}$

In a benchmark case, where subjects' payoffs depend only on how close their actions are to the fundamental, subjects put equal weights on both signals. With increasing importance of coordination in their payoff function, the relative weight attached to the public signal increases. However, these weights are significantly smaller than predicted by equilibrium theory.

Observed weights can be explained by limited levels of reasoning, where Level 1 is defined by the optimal action of a player who neglects that public signals provide more information about other players' beliefs, and Level 2 is the best response to Level 1 . The experiment reveals that for interior values of $r$, subjects' weights on the public signal are smaller or around the weights associated with Level $2{ }^{6}$ In the limiting case where subjects' payoffs depend only on the distance between their

\footnotetext{
${ }^{4}$ Morris et al. (2006) show that for correlated signals, the result by Morris and Shin (2002) may hold even when public signals are more precise than private signals. Myatt and Wallace (2014) also consider correlated private signals. They show that in a Lucas-Phelps island economy all signals should be provided with the same correlation coefficient.

${ }^{5}$ Appendix D compares the results for the different treatments with theoretical predictions from equilibrium theory and different levels of reasoning.

${ }^{6}$ Cornand and Heinemann (2014) did not observe learning effects, except for the case where $r=1$.
} 
actions ( $r=1$ ), subjects tend to apply higher levels of reasoning. However, they still put a positive weight on their private signals, which prevents full coordination. ${ }^{7}$

Models of cognitive hierarchies are based on the assumption that agents decide with limited levels of reasoning about decisions of others. Nagel (1995) and Stahl and Wilson (1994) define level-0 types as subjects who choose an action randomly from a uniform distribution over all possible actions. For $k>0$, a level- $k$ type is playing best response to Level $k-1$. Obviously, this definition hinges on the definition of the action space. While experiments typically have a bounded action space, the game by Morris and Shin (2002) allows for actions being reals. Hence level-0 types choose actions from a uniform distribution on the reals. If actions have a uniform distribution on the reals, expected losses from the coordination part of the payoff function are infinite for any action, so that any action is a best reply as long as $r>0$. However, expected losses resulting from deviations from the fundamental are minimized by choosing the expected state. Hence, Cornand and Heinemann (2014) define the best response to a uniform distribution over all reals as $a_{i}^{1}=E_{i}(\theta)$. Note that $a_{i}^{1}=E_{i}(\theta)$ is the best response of a player who ignores that public signals are shared with other players and it is also the welfare maximizing strategy combination. ${ }^{8}$

The action of a level-1 player is

$$
a_{i}^{1}=E_{i}(\theta)=\frac{\beta x_{i}+\alpha y}{\alpha+\beta}=\gamma_{1} x_{i}+\left(1-\gamma_{1}\right) y,
$$

where $\gamma_{1}=\beta /(\alpha+\beta)$ is the weight that a level-1 player attaches to the private signal.

Suppose that a player $j$ attaches weight $\gamma_{1}$ to her private signal. The best response to this is

$$
\begin{aligned}
a_{i}^{2} & =(1-r) E_{i}(\theta)+r E_{i}\left(a_{j}^{1}\right) \\
& =(1-r) E_{i}(\theta)+r \gamma_{1} E_{i}\left(x_{j}\right)+r\left(1-\gamma_{1}\right) y \\
& =\left(1-r\left(1-\gamma_{1}\right)\right) E_{i}(\theta)+r\left(1-\gamma_{1}\right) y .
\end{aligned}
$$

So, the action associated with level-2 reasoning is

$$
a_{i}^{2}=\frac{\alpha \beta(1-r)+\beta^{2}}{(\alpha+\beta)^{2}} x_{i}+\frac{\alpha^{2}+\alpha \beta(1+r)}{(\alpha+\beta)^{2}} y
$$

and the weight on private signals for a second level of reasoning is

$$
\gamma_{2}=\frac{\alpha \beta(1-r)+\beta^{2}}{(\alpha+\beta)^{2}}
$$

If all agents are level-2 players, the expected welfare is given by

\footnotetext{
${ }^{7}$ Equilibrium theory does not yield a unique prediction for $r=1$ : any coordinated strategy is an equilibrium. However, the limit of equilibria in games with increasing $r$ uniquely selects a strategy in which all agents follow the public signal and ignore all private information.

8 This definition follows Cornand and Heinemann (2014). In their experiment, choices were restricted to a bounded subset, allowing for an alternative notion of Level 0 which is, however, inconsistent with the evidence.
} 


$$
\begin{aligned}
E(W(a, \theta)) & =-E\left[\int_{i \in(0,1)}\left(a_{i}-\theta\right)^{2} d i\right]=-E\left[\int_{i \in(0,1)}\left(\gamma_{2} x_{i}+\left(1-\gamma_{2}\right) y-\theta\right)^{2} d i\right] \\
& =-\left(\gamma_{2}\right)^{2} \frac{1}{\beta}-\left(1-\gamma_{2}\right)^{2} \frac{1}{\alpha}=-\frac{(\alpha+\beta)^{2}+r^{2} \alpha \beta}{(\alpha+\beta)^{3}} .
\end{aligned}
$$

Taking derivatives and rearranging terms, we get

$$
\frac{\partial E(W)}{\partial \alpha}=\frac{\alpha^{2}+\left(2+r^{2}\right) \alpha \beta+\left(1-r^{2}\right) \beta^{2}}{(\alpha+\beta)^{4}}>0
$$

and

$$
\frac{\partial E(W)}{\partial \beta}=\frac{\left(1-r^{2}\right) \alpha^{2}+\left(2+r^{2}\right) \alpha \beta+\beta^{2}}{(\alpha+\beta)^{4}}>0 .
$$

Hence, the comparative statics exercise shows that increasing the precision of either type of information unambiguously increases welfare. This result is in stark contrast to Morris and Shin (2002), who show that increasing the precision of public signals may reduce welfare.

Result 1: In stark contrast to Morris and Shin's welfare analysis, we find that - when agents have a level of reasoning of degree 2 - increasing the precision of public information is always raising expected welfare.

This result still holds for any mixture of level- 1 and level-2 agents. ${ }^{9}$ Since expected welfare is independent from coordination failure and just depends on how close actions are to the fundamental, any information that yields a lower expected difference between actions and fundamentals, raises expected welfare. If an agent attaches a weight as prescribed by Level 1, Level 2, or anything in between, an increasing precision of signals reduces the expected deviation of her action from the fundamental. Hence, if all agents apply low levels (even different ones), increasing precision of information increases expected welfare.

With higher levels of reasoning, the relative weights on the two signals approach equilibrium weights, for which we know that public information can be welfare detrimental. This raises the question, at which level of reasoning such negative welfare effects start showing up. For higher levels of reasoning, the algebraic solution becomes untractable, so that we refer to simulations. For Level 3, we examine a wide range of parameters for which we determine the sign of the derivative of expected welfare with respect to the precision of public signals ${ }^{10}$. As shown in Figure 1, we detect parameter combinations with $r>0.6$ and a high ratio $\beta / \alpha$, for which an increase in the precision of public

9 If a fraction $\psi$ of agents applies Level 1 and the others Level 2, the expected welfare is $\psi E\left(W \mid \gamma_{1}\right)+(1-\psi) E\left(W \mid \gamma_{2}\right)$. Both expected-welfare terms are increasing in the precision of either signal. ${ }^{10}$ Appendix B determines the derivative of expected welfare with respect to the precision of public signals $\alpha$. 
signals $\alpha$ reduces expected welfare. ${ }^{11}$ Note, however, that Svensson's (2006) critique applies even more for limited levels of reasoning: if public information is withheld and agents employ level-3 reasoning, then private signals need to be more than 1.6 times as precise as the public signal for disclosure of the public signal to have a negative welfare effect.

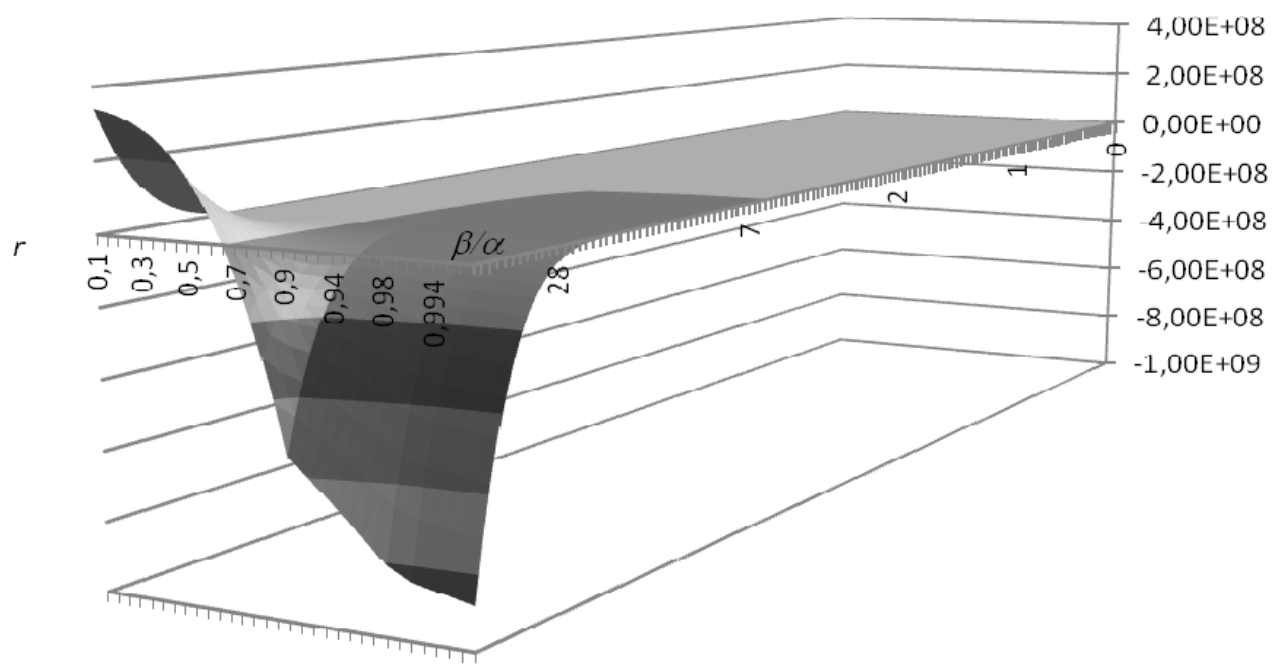

Figure 1 - Derivative of expected welfare with respect to the precision of public information $\alpha$ for level3 reasoning when the coordination part is a zero-sum game.

Whether increasing the precision of public information raises or lowers expected welfare if some agents apply Level 2 or a lower level and others apply Level 3 or a higher level, depends on the distribution of these types of players in the population. In Appendix E, we estimate the share of subjects who followed different levels of reasoning in the experiment of Cornand and Heinemann (2014). We show that for this distribution public information is welfare improving. Note however, that the gains from more precise public information to agents with Level 1 or 2 are small compared to the losses for high-level agents. Hence, if all players would be at least Level 1, even a small proportion of equilibrium players would be sufficient to cause detrimental welfare effects of public information.

It is a surprising result that negative welfare effects of public signals cannot occur when agents apply up to 2 levels of reasoning, but may be present for higher levels. In this paragraph, we shortly discuss what we view to be the intuitive reason behind this critical value. Level-2 players put a higher weight on public signals than justified by their relative precision, because they account for the additional payoff that they gain from predicting the likely actions of the other players. Thus, noisy

\footnotetext{
${ }^{11}$ Simulations are provided in the online appendix: http://www.macroeconomics.tu-berlin.de/fileadmin/fg124/heinemann/downloads/WLLR.xls.
} 
public signals drag the entire range of private actions away from the state of the world in direction of the public signal. However, the public signal also contributes to agents' forecasts of the state, which reduces the dispersion of actions around this state. For level-2 reasoning, the first effect can never outweigh the latter. By definition of Level 2, agents do not account for other agents to put a higher weight on the public signal, and thereby, the welfare detrimental effects of public information associated with strategic complementartities cannot unfold. Strategic behavior only kicks in, when agents also account for the higher weights of others, which requires more than 2 levels of reasoning. Then, each player's asymmetric response to the two signals induces other players to respond with an even higher weight on the public signal. Strategic complementarity affects behavior only from Level 3 onwards. This is because agents account for others' overreactions to public signals only from Level 3 onwards. Such higher-level reasoning seems to be a pre-requisite for public information to be welfare detrimental.

\section{4 - Welfare analysis for level-2 reasoning with coordination entering welfare}

If coordination is socially desirable, then public information is beneficial in equilibrium, while private information may have detrimental effects on welfare. This is an outcome, for example in Woodford's (2005) variant of the model, where coordination enters the welfare function. In this section, we check the robustness of these results with respect to limited levels of reasoning. The payoff for agent $i$ is given by

$$
u_{i}(a, \theta)=-(1-r)\left(a_{i}-\theta\right)^{2}-r \int_{0}^{1}\left(a_{i}-a_{j}\right)^{2} d j .
$$

Summing up individual utilities, social welfare is given by:

$$
W(a, \theta) \equiv \int_{0}^{1} u_{i}(a, \theta) d i=-(1-r) \int_{0}^{1}\left(a_{i}-\theta\right)^{2} d i-r \int_{0}^{1} \int_{0}^{1}\left(a_{i}-a_{j}\right)^{2} d j d i,
$$

where the last component accounts for socially desirable coordination. If agents attach weight $\gamma$ to private signals, expected welfare is

$$
\begin{aligned}
E(W(a, \theta)) & =-(1-r) E\left[\int_{i \in(0,1)}\left(\gamma\left(x_{i}-\theta\right)+(1-\gamma)(y-\theta)\right)^{2} d i\right]-r E\left[\int_{i \in(0,1)} \int_{j \in(0,1)}\left(\gamma\left(x_{i}-x_{j}\right)\right)^{2} d j d i\right] \\
& =-\gamma^{2} \frac{1+r}{\beta}-(1-\gamma)^{2} \frac{1-r}{\alpha} .
\end{aligned}
$$

Inserting the weight $\gamma_{2}$, associated with level-2 reasoning, the sign of $\partial E(W) / \partial \beta$ equals the sign of ${ }^{12}$

\footnotetext{
12 See proof in Appendix C.
} 


$$
(1+r)\left(1+\frac{\beta}{\alpha}\right)\left(1-r+\frac{\beta}{\alpha}\right)^{2}-2 r\left(1-r+\frac{\beta}{\alpha}+r \frac{\beta}{\alpha}\right)^{2} .
$$

Simulations as depicted in Figure 2, show that the sign of this term is ambiguous.

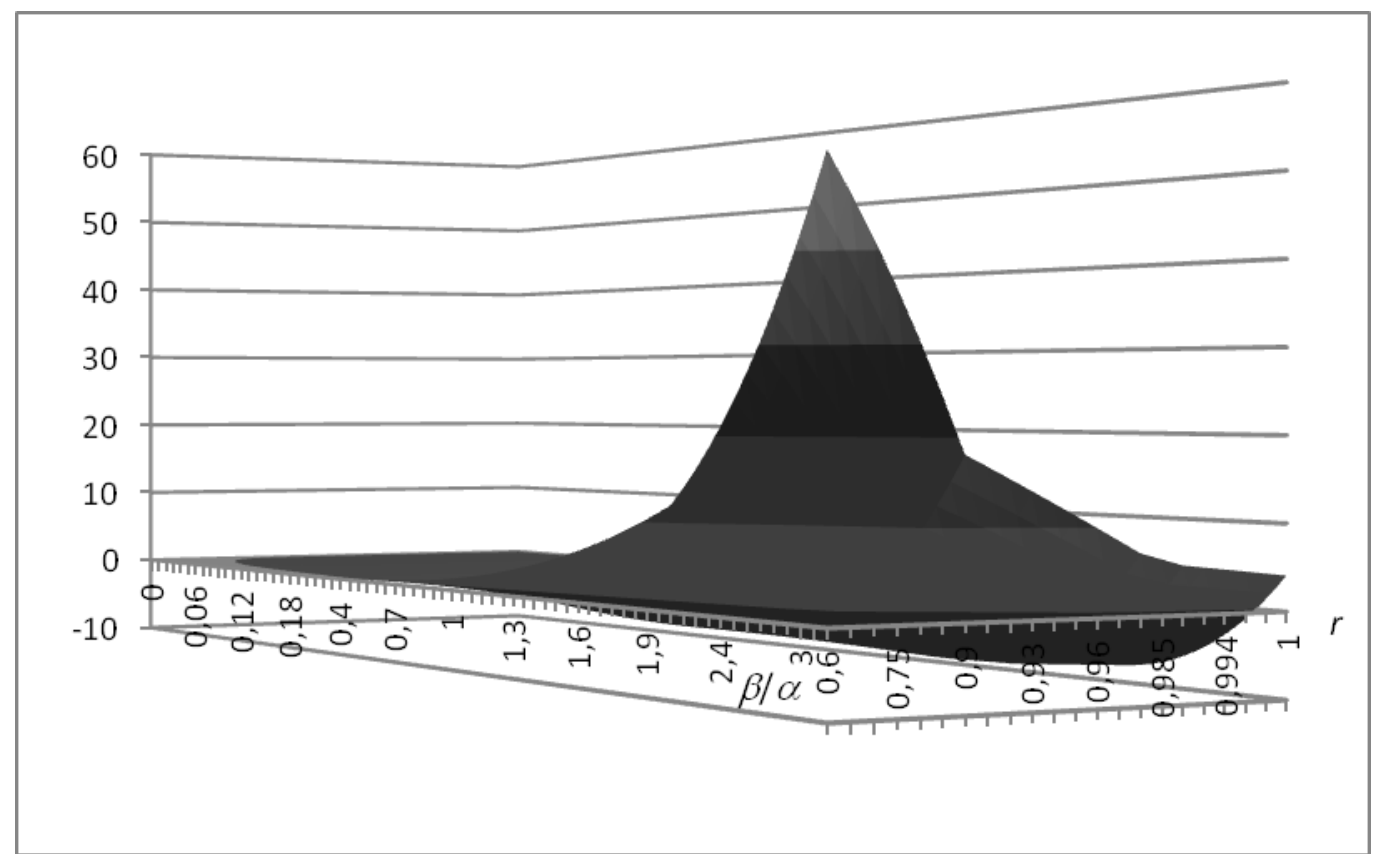

Figure 2 - Derivative of the expected welfare with respect to the precision of private information for level-2 reasoning in the game where coordination is socially useful.

More precisely, increasing the precision of private information can reduce welfare if $\beta / \alpha<3$ and $r>$ 0.6. ${ }^{13}$ The reason is that agents put a larger weight on private signals than is socially optimal. Higher precision of private signals raises the weight on private signals, which impedes desirable coordination. In the extreme case where $r \rightarrow 1$, providing subjects with private information would always be detrimental if they attach a positive weight to it. The experiment by Cornand and Heinemann (2014), but also Fehr et al. (2011) indicate that subjects are indeed responding to private signals in pure coordination games as for $r=1$.

A rising precision of public signals unambiguously raises expected welfare. As shown in Appendix C, this also holds for level-2 reasoning. Qualitatively, the result is the same as for fully rational agents. So, the advantage of transparency when coordination is socially desirable is robust to limited levels of reasoning.

Result 2: If coordination is socially desirable and agents apply limited levels of reasoning, increasing the precision of private information may reduce expected welfare, while an increasing precision of public information is always beneficial.

\footnotetext{
${ }^{13}$ Simulations are provided in the online appendix: http://www.macroeconomics.tu-berlin.de/fileadmin/fg124/heinemann/downloads/WLLR.xls.
} 


\section{5 - Limited levels of reasoning and partial publicity}

Concepts of intermediate publicity as in Cornand and Heinemann (2008), Morris et al. (2006), or Myatt and Wallace (2014) seem especially relevant for representing the way subjects deal with public signals. The model of Cornand and Heinemann (2008) is very similar to that of Morris and Shin (2002) with the same utility, distribution, and welfare functions. The difference is that agents have access to the public signal $y$ only with some probability $P .{ }^{14}$ In an application, Walsh (2007) has shown that such partial publicity may be especially valuable if cost shocks are serially correlated.

Cornand and Heinemann (2008) show that it is always better to provide public information with highest possible precision and eventually disclose this information only to some fraction of all agents than withholding public signals entirely. ${ }^{15}$ The optimal degree of publicity may be smaller than one even if an increasing precision of fully public signals has positive welfare effects. In particular, partial publicity may be optimal when public information is more precise than private signals. Thus, the result survives Svensson's (2006) critique. We now address the question, whether these results are robust to limited levels of reasoning.

Let us solve the Cornand and Heinemann model for two levels of reasoning. Again, the first level of reasoning for a player who receives both signals is given by the Bayesian expectation of $\theta$, so that the weight on the private signal with a first level of reasoning is $\gamma_{1}=\beta /(\alpha+\beta)$ and the corresponding action is $a_{j}=\gamma_{1} x_{j}+\left(1-\gamma_{1}\right) y$. The best response to this is

$$
\begin{aligned}
a_{i} & =(1-r) E_{i}(\theta)+r E_{i}\left(a_{j}\right) \\
& =(1-r) E_{i}(\theta)+r\left[P\left(\gamma_{1} E_{i}\left(x_{j}\right)+\left(1-\gamma_{1}\right) y\right)+(1-P) E_{i}\left(x_{j}\right)\right] \\
& =\frac{\alpha \beta(1-r P)+\beta^{2}}{(\alpha+\beta)^{2}} x_{i}+\frac{\alpha^{2}+\alpha \beta(1+r P)}{(\alpha+\beta)^{2}} y .
\end{aligned}
$$

Hence, the weight on private signals for the second level of reasoning is

$$
\gamma_{2}=\frac{\alpha \beta(1-r P)+\beta^{2}}{(\alpha+\beta)^{2}} .
$$

As Cornand and Heinemann (2008) have shown, the expected welfare for a degree of publicity $P$ is given by:

\footnotetext{
${ }^{14}$ Since there is a continuum of identical agents, the fraction of agents who receive public information equals $P$ almost certainly. Without loss of generality, agents $i \in[0, P]$ receive the public signal and agents $i \in(P, 1]$ rely on their private signals only. The signal $y$ is "public" in the sense that the actual realization of $y$ is common knowledge among agents $i \in[0, P]$.

${ }^{15}$ Baeriswyl and Cornand (2014) show that limiting the degree of publicity has the same welfare effects as providing information to all agents with idiosyncratic noise.
} 


$$
\begin{aligned}
E(W(a, \theta)) & =-E\left[\int_{i \in(0,1)}\left(a_{i}-\theta\right)^{2} d i\right] \\
& =-E\left[\int_{i \in(0, P)}\left(\gamma x_{i}+(1-\gamma) y-\theta\right)^{2} d i\right]-E\left[\int_{i \in(P, 1)}\left(x_{i}-\theta\right)^{2} d i\right] \\
& =-P\left[\gamma^{2} \frac{1}{\beta}+(1-\gamma)^{2} \frac{1}{\alpha}\right]-(1-P) \frac{1}{\beta},
\end{aligned}
$$

where $\gamma$ is the weight that agents put on the private signal. If agents use equilibrium strategies, the weight on the private signal is

$$
\gamma^{*}=\frac{\beta(1-r P)}{\alpha+\beta(1-r P)}
$$

and the resulting optimal degree of publicity is

$$
P^{*}=\min \left\{1, \frac{\alpha+\beta}{3 r \beta}\right\} \text {. }
$$

If, instead, agents use level-2 reasoning

$$
E(W(a, \theta))=-P \frac{(\alpha+\beta)^{2}+r^{2} P^{2} \alpha \beta}{(\alpha+\beta)^{3}}-(1-P) \frac{1}{\beta}
$$

and expected welfare changes with variations in the degree of publicity $P$ according to

$$
\begin{aligned}
& \frac{\partial E(W)}{\partial P}=\frac{-1}{\alpha+\beta}-3 P^{2} \frac{\alpha \beta r^{2}}{(\alpha+\beta)^{3}}+\frac{1}{\beta} . \\
& \frac{\partial E(W)}{\partial P} \geq 0 \Leftrightarrow P \leq \frac{\alpha+\beta}{\sqrt{3} \beta r} .
\end{aligned}
$$

Thus, for level-2 reasoning the optimal degree of publicity is

$$
P^{*}\left(\gamma_{2}\right)=\min \left\{1, \frac{\alpha+\beta}{\sqrt{3} r \beta}\right\} .
$$

While full transparency is optimal for Level 1, because agents choose the social optimum, the optimal degree of publicity may be smaller than 1 , if agents have higher levels of reasoning. In this respect, the qualitative result by Cornand and Heinemann (2008) is robust. However, optimal publicity is larger than in an economy where agents use equilibrium strategies. Furthermore, for $P^{*}\left(\gamma_{2}\right)$ to be smaller than 1 , we need $\alpha / \beta<\sqrt{3} r-1<0.74$. Thus, contrary to the result by Cornand and Heinemann (2008), accounting for limited levels of reasoning makes the optimal degree of publicity subject to Svensson's (2006) critique: with level-2 reasoning, public information needs to be less precise than private information to obtain an interior optimal degree of publicity. Thus, we can state our third result. 
Result 3: when agents have a level of reasoning of degree 2-the optimal degree of publicity may be smaller than 1. In contrast to the conclusion from the equilibrium analysis in Cornand and Heinemann (2008), public information needs to be sufficiently less precise than private information to justify limited publicity.

The intuition behind this result is that for any given degree of publicity, increasing levels of reasoning raises the weight that agents put on the public signal. Thereby, the welfare detrimental effects of public information are getting more important. To balance this, the degree of publicity should be lower.

\section{6 - Welfare effects of transparency with policy interventions and limited levels of reasoning}

James and Lawler (2011) show that opacity is always optimal when the central bank simultaneously releases a public signal and uses a policy instrument for stabilization policy. The reason is that public policy and private actions are perfect substitutes in responding to the fundamental (shock). In the firstbest solution, the sum of an agents' private and the public action ${ }^{16}$ should equal the expected state of the world given this agent's private and the government's information. By disclosing its information to private agents, the government induces a distortion in private actions towards this public signal. If the government would act after private agents, it could undo the bias and transparency would have no welfare effect. With simultaneous decisions, however, private agents take the government's equilibrium action into account and deviate even further from their private signals. Thereby, the sum of both actions gets distorted away from the optimum. In order to avoid this, the government should secretely respond to its information without disclosing or signaling it to the private sector. This logic is robust to coordination being socially desirable and to the possibility of providing partially public information (James and Lawler, 2012). As we formally show in this section, it also holds when private agents choose actions with limited levels of reasoning. The conclusions by James and Lawler just require that private responses to public signals differ from the social optimum, which is true under limited levels of reasoning of any degree except 1.

In their model, there is a policy maker and a continuum of private sector agents, indexed by $i$ and uniformly distributed over the unit interval. Agent $i$ chooses action $a_{i} \in \mathrm{R}$ to maximize a utility function:

$$
u_{i}=-(1-r)\left(a_{i}-\theta-g\right)^{2}-r\left(L_{i}-\bar{L}\right)
$$

\footnotetext{
${ }^{16}$ Actually, it is the difference, because James and Lawler (2011) define private and policy actions with opposite signs.
} 
where $\theta$ is the fundamental state of the economy, $g$ is the policy makers' instrument, $r$ is a constant, such that $0 \leq r<1, \quad L_{i} \equiv \int_{0}^{1}\left(a_{j}-a_{i}\right)^{2} d j$, and $\bar{L} \equiv \int_{0}^{1} L_{j} d j$. Both the policy maker and agents are uncertain about the true state $\theta$. Prior to deciding on $g$, the policy maker observes a noisy signal $z$ $z=\theta+\phi$, with $\phi \sim \mathrm{N}\left(0, \sigma_{\phi}^{2}\right)$.

The signal that the policy maker discloses to the public is

$$
y=\theta+\phi+\xi \text {, with } \xi \sim \mathrm{N}\left(0, \sigma_{\xi}^{2}\right) .
$$

Additionally to receiving the public signal $y$, agents also observe their own idiosyncratic noisy signals:

$$
x_{i}=\theta+\varepsilon_{l} \text {, with } \varepsilon_{l} \sim \mathrm{N}\left(0, \sigma_{\varepsilon}^{2}\right) .
$$

All noise terms are independent of $\theta$, and independent of each other. James and Lawler assume that the policy maker follows the rule:

$$
g=\rho z
$$

With such informational structure, private agents' expectations are given by

$$
E_{i}(\theta)=\frac{\left(\sigma_{\phi}^{2}+\sigma_{\xi}^{2}\right) x_{i}+\sigma_{\varepsilon}^{2} y}{\Sigma}=E_{i}\left(x_{j}\right) \text { and } E_{i}(z)=\frac{\left(\sigma_{\varepsilon}^{2}+\sigma_{\phi}^{2}\right) y+\sigma_{\xi}^{2} x_{i}}{\Sigma} \text {, where } \Sigma=\sigma_{\varepsilon}^{2}+\sigma_{\phi}^{2}+\sigma_{\xi}^{2} \text {. }
$$

The action of a level-1 player is

$$
a_{i}^{1}=E_{i}(\theta+g)=\frac{\sigma_{\phi}^{2}+(1+\rho) \sigma_{\xi}^{2}}{\Sigma} x_{i}+\frac{\sigma_{\varepsilon}^{2}+\rho\left(\sigma_{\varepsilon}^{2}+\sigma_{\phi}^{2}\right)}{\Sigma} y=\gamma_{1 x} x_{i}+\gamma_{1 y} y .
$$

Note that $\gamma_{1 x}+\gamma_{1 y}=1+\rho$. The policy parameter $\rho$ will turn out to be negative. The best response to $a_{i}^{1}$ (which is the action of a level-2 player) is:

$$
\begin{aligned}
a_{i}^{2} & =(1-r) E_{i}(\theta+g)+r E_{i}\left(a_{j}^{1}\right) \\
& =(1-r) E_{i}(\theta)+(1-r) \rho E_{i}(z)+r\left(\gamma_{1 x} E_{i}\left(x_{j}\right)+\gamma_{1 y} y\right)=\gamma_{2 x} x_{i}+\gamma_{2 y} y,
\end{aligned}
$$

with

$$
\begin{aligned}
& \gamma_{2 x}=\frac{1}{\Sigma}\left[\left(1-r \frac{\sigma_{\varepsilon}^{2}-\rho \sigma_{\xi}^{2}}{\Sigma}\right)\left(\sigma_{\phi}^{2}+\sigma_{\xi}^{2}\right)+(1-r) \rho \sigma_{\xi}^{2}\right], \\
& \gamma_{2 y}=\frac{1}{\Sigma}\left[\left(1-r \frac{\sigma_{\varepsilon}^{2}-\rho \sigma_{\xi}^{2}}{\Sigma}\right) \sigma_{\varepsilon}^{2}+(\rho+r) \sigma_{\varepsilon}^{2}+\rho \sigma_{\phi}^{2}\right] .
\end{aligned}
$$

In this framework, expected welfare is given by 


$$
\begin{aligned}
& E(W(a, \theta))=-E\left[\int_{i \in(0,1)}\left(a_{i}-\theta-g\right)^{2} d i\right]=-E\left[\int_{i \in(0,1)}\left(\gamma_{2 x} x_{i}+\gamma_{2 y} y-\theta-\rho z\right)^{2} d i\right] \\
& =-E\left[\int_{i \in(0,1)}\left(\gamma_{2 x} \varepsilon_{i}+\gamma_{2 y}(\phi+\xi)-\rho \phi\right)^{2} d i\right]=-\left(\gamma_{2 x}\right)^{2} \sigma_{\varepsilon}^{2}-\left(\gamma_{2 y}-\rho\right)^{2} \sigma_{\phi}^{2}-\left(\gamma_{2 y}\right)^{2} \sigma_{\xi}^{2} .
\end{aligned}
$$

To derive the optimal communication strategy given the optimal policy, we need to study the sign of the derivative of the expected welfare with respect to $\sigma_{\xi}^{2}$ at the optimal stabilization policy $\rho^{*}$. Inserting level-2 weights in the expected welfare function and solving its first-order condition yields the optimal policy parameter

$$
\rho^{*}=-\frac{\Sigma^{2} \sigma_{\varepsilon}^{2}+r^{2}\left(\sigma_{\phi}^{2}+\sigma_{\xi}^{2}\right) \sigma_{\varepsilon}^{4}}{\Sigma^{2}\left(\sigma_{\varepsilon}^{2}+\sigma_{\phi}^{2}\right)+r^{2} \sigma_{\varepsilon}^{4} \sigma_{\xi}^{2}} .
$$

The resulting expected welfare is given by

$$
E(W(a, \theta))=\frac{-\sigma_{\varepsilon}^{2} \sigma_{\phi}^{2}\left[\Sigma^{2}+r^{2} \sigma_{\varepsilon}^{2}\left(\sigma_{\phi}^{2}+\sigma_{\xi}^{2}\right)\right]}{\left[\Sigma^{2}\left(\sigma_{\varepsilon}^{2}+\sigma_{\phi}^{2}\right)+r^{2} \sigma_{\varepsilon}^{4} \sigma_{\xi}^{2}\right]} .
$$

The derivative of this expression with respect to the variance of public information is

$$
\left.\frac{d E(W(a, \theta))}{d \sigma_{\xi}^{2}}\right|_{\rho=\rho^{*}}=\frac{r^{2} \sigma_{\varepsilon}^{4} \sigma_{\phi}^{4}\left(\Sigma^{2}+r^{2} \sigma_{\varepsilon}^{4}\right)}{\left[\Sigma^{2}\left(\sigma_{\varepsilon}^{2}+\sigma_{\phi}^{2}\right)+r^{2} \sigma_{\varepsilon}^{4} \sigma_{\xi}^{2}\right]^{2}}>0 .
$$

This derivative is unambiguously positive, which indicates that the expected welfare increases with rising variance of public information. As in the original contribution by James and Lawler (2011), optimal policy calls for the government secretely responding to its information about the shock by its policy variable. Without any public information, private agents are left to respond to their private signals only. Recall that $\gamma_{1 x}+\gamma_{1 y}+|\rho|=1$. The optimal response of the government equals the relative precision of its information compared to the precision of private signals, while private agents respond to their signals with a factor that equals the relative precision of their signals. In effect, $a_{i}-g=E\left(\theta \mid x_{i}, z\right)$, which is the optimal response to a private agent's available information that a social planner would implement.

Result 4: As in James and Lawler (2011), social welfare is unambiguously decreasing with rising precision of public signals when agents have limited levels of reasoning of degree 2.

\section{7 - Conclusion}

The literature in the vein of Morris and Shin (2002) has largely been interpreted in terms of central bank communication and concentrates on discussing welfare effects of central bank announcements 
using equilibrium theory. We contribute to this discussion by showing that deviations from full rationality that are regularly observed in experiments relying on generalized beauty contest games as Morris and Shin (2002), provide an additional argument in favor of transparency. Different from all the previous papers, our argument is not based on equilibrium theory and proposes instead a behavioral approach. Subjects in the lab behave more in line with limited levels of reasoning than with equilibrium theory. Under limited levels of reasoning, subjects attach smaller weights to public information than in equilibrium. This at least reduces negative welfare effects of public information. The precise conclusions from such a behavioristic view depend on the environment.

If coordination is socially desirable, transparency is beneficial in equilibrium as has been shown by e.g. Hellwig (2005), Woodford (2005), and Roca (2010). Limited levels of reasoning do not alter this result for public signals. Our simulations show, however, that private information may have negative welfare effects under limited levels of reasoning.

If welfare just depends on the distance between private actions and a fundamental, negative welfare effects of public signals are possible in equilibrium (Morris and Shin, 2002). Svensson (2006) argued that this requires public information to be less precise than private signals, which is unlikely given the empirical evidence. With levels of reasoning up to Level 2, as observed in experiments, public information cannot have negative welfare effects in this standard model. For higher levels of reasoning, negative effects are possible but require an even lower precision of public signals.

While the standard model assumes uncorrelated signal errors, Morris et al. (2006) have shown that for correlated signals, public information may reduce welfare, even if it is more precise than private signals. Here, the reason is that the public signal provides an additional hint on agents' beliefs and will be used with a larger weight than an uncorrelated public signal. Since agents with limited levels of reasoning put lower weights on public signals than in equilibrium, the negative welfare effects are attenuated.

Cornand and Heinemann (2008) emphasized that negative welfare effects of public signals can be better addressed by limited publicity than by withholding information entirely. Disclosing information to a subset of market participants yields a higher welfare than full disclosure because it reduces the degree of common knowledge responsible for overreaction to public information. Baeriswyl and Cornand (2014) show that the same welfare can be attained by disclosing public information with what they call a limited degree of transparency, i.e. by adding idiosyncratic noise to the receivers' signals. This turns public information to a hybrid between public and private signals. Under limited levels of reasoning, the optimal degree of publicity is higher than in equilibrium and for level-2 reasoning, a limited degree of publicity can be optimal only if public information is less precise than private signals. Due to the equivalence of both approaches, the same conclusion holds for a limited degree of transparency.

When the central bank discloses information, it exerts an influence on market expectations; at the same time, it collects information from markets to assess the economic situation and make 
decisions. In such an environment of endogenous information, transparency affects the quality of the central banks' own information. Amato and Shin (2006) and Morris and Shin (2005) show that the informative value of prices decreases with the degree of strategic complementarities. Baeriswyl (2011) shows that here, the optimal degree of transparency is lower than for exogenous information and does not depend on the precision of private signals. Since limited levels of reasoning reduce the weight that private agents put on public signals, they also reduce the feedback problem associated with endogenous information.

If the policy maker can take actions that are perfect substitutes to private actions as in the model by James and Lawler (2011), then private agents need only respond to their private signals and transparency can just have negative welfare effects. Here, the policy maker should respond to its own information as secretely as possible and avoid disclosing or signalling its information to the public. This conclusion is not affected by limited levels of reasoning.

If policy interventions can be observed by the private sector, they will also be used as signals about the central bank's own information. Baeriswyl and Cornand (2010) provide an example, in which the central bank deliberately distorts its response to labor supply and mark-up shocks in order to optimally balance the direct stabilizing role of its instrument and its indirect effect through shaping firms' beliefs. Extracting the information contained in such policy actions requires a high degree of strategic sophistication. Subjects in experiments are usually not sufficiently sophisticated to extract this information correctly. In addition, rational signal extraction requires confidence in the rationality of the policy maker. Any doubt in the rationality of the decision process lowers the perceived precision of the indirectly revealed information. Once again, the behavioral approach would lead to an attenuation of the potential to reduce welfare by public signals.

A recent paper by Baeriswyl and Cornand (2015) presents an experiment similar to the experiment in Cornand and Heinemann (2014) with the crucial difference that subjects receive public and private signals of different precision. On top of choosing actions in the beauty contest game, subjects also state their expectation on the fundamental. In these expectations, subjects put a higher weight on the more precise signal, but, this weight is smaller than the Bayesian weight. Thereby, subjects' expectations of the fundamental are biased towards the less precise signal. Actions in the beauty contest game are best described by limited levels of reasoning when Level 1 is defined as the biased first-order expectation on the fundamental. Thus, there are two behavioral biases that may go in the same or in opposite directions depending on whether private or public information is more accurate. When the public signal is less precise than the private signal, the two effects may cancel each other and lead to behavior that cannot be distinguished from equilibrium, as was observed in one of the treatments in this experiment. Here, transparency may exacerbate the overweighting of public information already present in the expectation of the fundamental and may thus impair welfare. If the public signal is more precise than the private signal, biased first-order expectations reduce the weight of the public signal even more than ordinary levels of reasoning (compared to equilibrium) and public 
information is even less likely to have negative welfare effects. Here, the overreaction to public signals is even beneficial, because it helps setting off the bias in first-order expectations.

In equilibrium theory, negative welfare effects of transparency require that strategic complementarities be sufficiently strong, the private values of coordination be sufficiently higher than the social value of coordination, and signals provided by the central bank be of a low precision compared to private signals or highly correlated with them. Endogeneity of public information or private signals being extracted from observed policy actions raise the likelihood that transparency has negative effects. In all of these cases, limited levels of reasoning mitigate potential negative welfare effects of transparency.

Experiments, such as Nagel (1995) or Cornand and Heinemann (2014) indicate that actual behavior in beauty contest games can be better described by a cognitive hierarchy model with level-2 reasoning. In stark contrast to equilibrium analyses, we find that - when agents have a level of reasoning of degree 2 - increasing the precision of public information always raises expected welfare in the standard beauty contest game and even partial publicity can improve expected welfare only if public signals are less precise than private information. Accounting for limited levels of reasoning thus provides some new insights about the welfare effects of central bank transparency.

\section{References}

Amato, J., and H.S. Shin (2006), "Imperfect Common Knowledge and the Information Value of Prices," Economic Theory, 27, 213-241.

Angeletos, G.-M., and A. Pavan (2007a), "Socially optimal coordination: Characterization and policy implications," Journal of the European Economic Association (Papers and Proceedings), 5, 585593.

Angeletos, G.-M., and A. Pavan (2007b), "Efficient Use of Information and Social Value of Information," Econometrica, 75, 1103-1142.

Baeriswyl, R. (2011), "Endogenous Central Bank Information and the Optimal Degree of Transparency,” International Journal of Central Banking, 7, 85-111.

Baeriswyl, R., and C. Cornand (2010), “The Signaling Role of Policy Actions”, Journal of Monetary Economics, 57(6), pp. 682-695, 2010.

Baeriswyl, R., and C. Cornand (2014), "Reducing Overreaction to Central Banks' Disclosures : Theory and Experiment," Journal of the European Economic Association, 12, 1087-1126.

Baeriswyl, R., and C. Cornand (2015), “The Predominant Role of Signals’ Precision”, mimeo.

Cornand, C., and F. Heinemann (2008), “Optimal Degree of Public Information Dissemination,” The Economic Journal, 118, 718-742.

Cornand, C., and F. Heinemann (2014), "Measuring Agents' Reaction to Private and Public Information in Games with Strategic Complementarities”, Experimental Economics, 17(1), 61-77.

Fehr, D., F. Heinemann, and A. Llorente-Saguer (2011), "The Power of Sunspots: an Experimental Analysis,” SFB 649, Discussion Paper 2011-070.

Hellwig, C. (2005), "Heterogeneous Information and the Welfare Effects of Public Information Disclosures,” UCLA, mimeo. 
James J., and P. Lawler (2011), "Optimal Policy Intervention and the Social Value of Public Information,” American Economic Review, 101, 1561-1574.

James J., and P. Lawler (2012), "Strategic Complementarity, Stabilization Policy, and the Optimal Degree of Publicity,” Journal of Money, Credit and Banking, 44, 551-572.

Kübler, D., and G. Weizsäcker (2004), "Limited Depth of Reasoning and Failure of Cascade Formation in the Laboratory," Review of Economic Studies, 71, 425-442.

Lorenzoni, G. (2010), "Optimal Monetary Policy with Uncertain Fundamentals and Dispersed Information,” Review of Economic Studies, 77, 305-338.

Morris, S., and H.S. Shin (2002), "Social Value of Public Information,” American Economic Review, 92, 1522-1534.

Morris, S., and H.S. Shin (2005), "Central Bank Transparency and the Signal Value of Prices", Brookings Papers on Economic Activity, 2, 1-66.

Morris, S., H. S. Shin, and H. Tong (2006), "Social Value of Public Information: Morris and Shin (2002) Is Actually Pro-Transparency, Not Con: Reply,” American Economic Review, 96, 453-55.

Myatt, D.P., and C. Wallace (2014), "Central Bank Communication Design in a Lucas-Phelps Economy,” Journal of Monetary Economics, 63, 64-79.

Nagel, R. (1995), “Unraveling in Guessing Games: An Experimental Study,” American Economic Review, 85, 1313-1326.

Roca, M. (2010), “Transparency and Monetary Policy with Imperfect Common Knowledge,” IMF Working Paper 10/91.

Romer, C.D., and D.H. Romer (2000), "Federal Reserve Private Information and the Behavior of Interest Rates," American Economic Review, 90, 429-457.

Shapiro, D., X. Shi, and A. Zillante (2014), "Level-k reasoning in a generalized beauty contest," Games and Economic Behavior, 86, 308-329.

Stahl, D. O., and P.W. Wilson (1994), "Experimental Evidence on Players' Models of Other Players," Journal of Economic Behavior and Organization, 25, 309-327.

Svensson, L.E.O. (2006), "Social Value of Public Information: Comment: Morris and Shin (2002) Is Actually Pro-Transparency, Not Con,” American Economic Review, 96, 448-452.

Walsh, C., (2007), “Optimal Economic Transparency,” International Journal of Central Banking, 3, 535.

Woodford, M. (2005), “Central Bank Communication and Policy Effectiveness,” in: The Greenspan Era: Lessons for the Future, Kansas City: Federal Reserve Bank of Kansas City, 399-474. 


\section{Appendix}

\section{A. Experimental design of Cornand and Heinemann (2014)}

For each pair of subjects, a fundamental state $\theta$ was drawn randomly using a uniform distribution from the interval $[50,450]$ and each subject received a private signal and each pair of subjects received a common (public) signal. Signals were drawn independently with a uniform distribution from $[\theta-10, \theta+10]$. In the first three stages, each subject had to decide on an action $a_{i}$ conditional on her signals. In the first stage (" $r=0$ "), played for 5 rounds, the payoff function was given by $100-\left(a_{i}-\theta\right)^{2}$. Here, the rational choice is $a_{i}=\left(x_{i}+y\right) / 2$. In the second stage (“ $r=1$ ”), played for 10 rounds, the payoff function was given by $100-\left(a_{i}-a_{j}\right)^{2}$, where $a_{j}$ denotes the partner's action. Here, any strategy that maps the public signal into the reals is an equilibrium, if both subjects coordinate on this strategy. The third stage, played for 30 rounds, treatments differed between sessions. There were six sessions for each of the three treatments. In Treatment " $r=0.5$ ”, the payoff function was $200-\left(a_{i}-a_{j}\right)^{2}-\left(a_{i}-\theta\right)^{2}$, while it was $400-\left(a_{i}-a_{j}\right)^{2}-3\left(a_{i}-\theta\right)^{2}$ in Treatment “ $r=0.25$ ” and $400-3\left(a_{i}-a_{j}\right)^{2}-\left(a_{i}-\theta\right)^{2}$ in Treatment " $r=0.75$ ". These three treatments correspond to the two-player version of the game by Morris and Shin (2002) and the equilibrium weight on the private signal is $(1-r) /(2-r)$. Cornand and Heinemann (2014) used the average weight on the private signal within a session as an independent observation. In the fourth stage, each subject was asked to state his expectations on the fundamental and his expectation regarding his partner's expectation of the fundamental.

B. Derivative of expected welfare with respect to the precision of the public signal for level-3 reasoning without coordination entering welfare

The expected welfare is increasing with rising $\alpha$ if and only if $\left[1+(2+r) s+\left(1+r+r^{2}\right) s^{2}\right]^{2}(1+s)+2\left[-r-2 r s-r^{2} s-r s^{2}-r^{2} s^{2}\right]\left[-r s-2 r^{2} s^{2}+r s^{3}+r^{2} s^{3}+s+2 s^{2}+s^{3}\right]>0$, where $s=\beta / \alpha$.

Proof:

We have: 


$$
\begin{aligned}
& E(W)=\frac{-\gamma^{2}}{\beta}-\frac{(1-\gamma)^{2}}{\alpha}, \\
& \gamma_{3}=\frac{(\alpha+\beta)^{2}-r \alpha^{2}-r(1+r) \alpha \beta}{(\alpha+\beta)^{3}} \beta, \\
& 1-\gamma_{3}=\frac{\alpha^{2}+(2+r) \alpha \beta+(1+r(1+r)) \beta^{2}}{(\alpha+\beta)^{3}} \alpha, \\
& \frac{\partial \gamma_{3}}{\partial \alpha}=\frac{(\alpha+\beta)[2(\alpha+\beta)-2 r \alpha-r(1+r) \beta] \beta-3\left[(\alpha+\beta)^{2}-r \alpha^{2}-r(1+r) \alpha \beta\right] \beta}{(\alpha+\beta)^{4}} .
\end{aligned}
$$

Replacing $\beta$ by $\alpha s$, we obtain:

$$
\begin{aligned}
& \frac{\gamma_{3}}{\beta}=\frac{(1+s)^{2}-r-\left(r+r^{2}\right) s}{\alpha(1+s)^{3}}, \\
& \frac{1-\gamma_{3}}{\alpha}=\frac{1+(2+r) s+\left(1+r+r^{2}\right) s^{2}}{\alpha(1+s)^{3}}, \\
& \frac{\partial \gamma_{3}}{\partial \alpha}=\frac{(1+s)[2(1+s)-2 r-r(1+r) s] s-3\left[(s+s)^{2}-r-r(1+r) s\right] s}{\alpha(1+s)^{4}} \\
& \quad=\frac{r s+2 r^{2} s^{2}-r s^{3}-r^{2} s^{3}+s-2 s^{2}-s^{3}}{\alpha(1+s)^{4}} .
\end{aligned}
$$

We can then calculate the derivative of the expected welfare with respect to $\alpha$, as a function of $\alpha, s$ and $r$ :

$$
\begin{aligned}
\frac{\partial E W}{\partial \alpha} & =\frac{(1-\gamma)^{2}}{\alpha^{2}}-\left[\frac{2 \gamma}{\beta}-\frac{2(1-\gamma)}{\alpha}\right] \frac{\partial \gamma}{\partial \alpha} \\
& =\frac{\left[1+(2+r) s+\left(1+r+r^{2}\right) s^{2}\right]^{2}}{\alpha^{2}(1+s)^{6}}-2 \frac{(1+s)^{2}-r-r s-r^{2} s-\left[1+(2+r) s+\left(1+r+r^{2}\right) s\right]}{\alpha(1+s)^{3}} \frac{\partial \gamma}{\partial \alpha} \\
& =\frac{1}{\alpha^{2}(1+s)^{7}}\left[\begin{array}{l}
\left.\left[1+(2+r) s+\left(1+r+r^{2}\right) s^{2}\right]^{2}(1+s)+2\left[-r-2 r s-r^{2} s-r s^{2}-r^{2} s^{2}\right] \cdot\right] \\
\cdot\left[-r s-2 r^{2} s^{2}+r s^{3}+r^{2} s^{3}+s+2 s^{2}+s^{3}\right]
\end{array}\right.
\end{aligned}
$$

C. Derivatives of expected welfare with respect to the precisions of private and public information for level-2 reasoning with coordination entering welfare

The expected welfare is always increasing in the precision of public information $\alpha$. It is increasing in the precision of private information $\beta$ if and only if

$$
(1+r)(1+s)[1-r+s]^{2}-2 r[1-r+s+r s]^{2}>0,
$$

where $s=\beta / \alpha$.

Proof: 
We have:

$$
\begin{aligned}
& E(W)=\frac{-(1+r) \gamma^{2}}{\beta}-\frac{(1-r)(1-\gamma)^{2}}{\alpha}, \\
& \gamma_{2}=\frac{\alpha \beta(1-r)+\beta^{2}}{(\alpha+\beta)^{2}}, \\
& 1-\gamma_{2}=\frac{\alpha^{2}+(1+r) \alpha \beta}{(\alpha+\beta)^{2}}, \\
& \frac{\partial \gamma_{2}}{\partial \alpha}=\frac{(\alpha+\beta) \beta(1-r)-2\left[\alpha \beta(1-r)+\beta^{2}\right]}{(\alpha+\beta)^{3}}, \\
& \frac{\partial \gamma_{2}}{\partial \beta}=\frac{(\alpha+\beta)[\alpha(1-r)+2 \beta]-2\left[\alpha \beta(1-r)+\beta^{2}\right]}{(\alpha+\beta)^{3}} .
\end{aligned}
$$

Replacing $\beta$ by $\alpha s$ :

$$
\begin{aligned}
& \frac{\gamma_{2}}{\beta}=\frac{1-r+s}{\alpha(1+s)^{2}}, \\
& \frac{1-\gamma_{2}}{\alpha}=\frac{1+s+r s}{\alpha(1+s)^{2}}, \\
& \frac{\partial \gamma_{2}}{\partial \alpha}=\frac{(1+s) s(1-r)-2\left[s(1-r)+s^{2}\right]}{\alpha(1+s)^{3}}=\frac{-s^{2}(1+r)-s(1-r)}{\alpha(1+s)^{3}}, \\
& \frac{\partial \gamma_{2}}{\partial \beta}=\frac{(1+s)[1-r+2 s]-2\left[s(1-r)+s^{2}\right]}{\alpha(1+s)^{3}}=\frac{1-r+s+r s}{\alpha(1+s)^{3}} .
\end{aligned}
$$

We can then calculate the derivatives of the expected welfare with respect to $\alpha$ and $\beta$, as functions of $\alpha, s$ and $r$ :

$$
\begin{aligned}
\frac{\partial E W}{\partial \alpha} & =\frac{(1-r)(1-\gamma)^{2}}{\alpha^{2}}-\left[\frac{2(1+r) \gamma}{\beta}-\frac{2(1-r)(1-\gamma)}{\alpha}\right] \frac{\partial \gamma}{\partial \alpha} \\
& =\frac{(1-r)[1+s+r s]^{2}}{\alpha^{2}(1+s)^{4}}+2 \frac{[(1+r)(1-r+s)-(1-r)(1+s+r s)] \cdot\left[s^{2}(1+r)+s(1-r)\right]}{\alpha^{2}(1+s)^{5}} \\
& =\frac{1}{\alpha^{2}(1+s)^{5}}\left[(1-r)(1+s)[1+s+r s]^{2}-2[(1+r)(1-r+s)-(1-r)(1+s+r s)] \cdot\left[s^{2}(1+r)+s(1-r)\right]\right] \\
& =\frac{1}{\alpha^{2}(1+s)^{5}}\left[(1-r)(1+s)[1+s+r s]^{2}+2 r[1-r+s+r s] \cdot\left[s^{2}(1+r)+s(1-r)\right]\right]>0, \\
\frac{\partial E(W)}{\partial \beta} & =\frac{(1+r) \gamma^{2}}{\beta^{2}}-\left[\frac{2(1+r) \gamma}{\beta}-\frac{2(1-r)(1-\gamma)}{\alpha}\right] \frac{\partial \gamma}{\partial \beta}
\end{aligned}
$$




$$
\begin{aligned}
& =\frac{(1+r)[1-r+s]^{2}}{\alpha^{2}(1+s)^{4}}-2 \frac{[(1+r)(1-r+s)-(1-r)(1+s+r s)] \cdot[1-r+s+r s]}{\alpha^{2}(1+s)^{5}} \\
& =\frac{(1+r)[1-r+s]^{2}}{\alpha^{2}(1+s)^{4}}-2 \frac{[(1+r)(1-r+s)-(1-r)(1+s+r s)] \cdot[1-r+s+r s]}{\alpha^{2}(1+s)^{5}} \\
& =\frac{1}{\alpha^{2}(1+s)^{5}}\left[(1+r)(1+s)[1-r+s]^{2}-2[(1+r)(1-r+s)-(1-r)(1+s+r s)] \cdot[1-r+s+r s]\right] \\
& =\frac{1}{\alpha^{2}(1+s)^{5}}\left[(1+r)(1+s)[1-r+s]^{2}-2 r[1-r+s+r s]^{2}\right] .
\end{aligned}
$$

\section{Limited levels of reasoning in the lab (Cornand and Heinemann, 2014)}

Table A summarizes the weights on the private signal for increasing levels of reasoning and the equilibrium weight on the private signal for different values of $r$. It also provides average observed weights (for the last half of rounds) in the experiment by Cornand and Heinemann (2014).

\begin{tabular}{llllllll}
\hline Value of $r$ & Level 1 & Level 2 & Level 3 & Level 4 & $\ldots$ & $\begin{array}{l}\text { Average observed } \\
\text { weight }\end{array}$ & $\begin{array}{l}\text { Equilibrium weight } \\
\text { (infinite levels of reasoning) }\end{array}$ \\
\hline 0 & & & & & & &
\end{tabular}

Table A - Theoretical and observed weight on $x_{i}$ depending on $r$ and on the level of reasoning

For interior values of $r$, most estimated weights on private signals are close to or above the weights given by level-2 reasoning. In treatments with $r=0.5$ and $r=0.25$, weights on the private signal are usually higher than the weights from level-2 reasoning. The hypothesis that weights equal those from level-2 reasoning is rejected in these treatments. In the treatment with $r=0.75$, weights are distributed around the weight from Level 2. Higher levels can be rejected by one-sided tests at $5 \%$. In the treatment with $r=1$, the weights tend to converge towards equilibrium, even though convergence is not settled within ten rounds. "These inhomogeneous test results indicate that subjects might behave in accordance to higher levels of reasoning with increasing r." (Cornand and Heinemann 2014, p. 74)

\section{E. Welfare effects of public information with heterogeneous strategies}

The theoretical analysis in the main text assumes that all agents employ the same strategy. The experiment by Cornand and Heinemann (2014), however, reveals that subjects do not converge to one strategy, but rather employ different strategies throughout the experiment. In particular, some subjects' strategies are close to equilibrium or put an even higher weight on public signals. Such a mixture of strategies may result in public information reducing expected welfare. This depends on the share of 
agents employing these different strategies. To deal with welfare effects under heterogeneity, we use the data of Cornand and Heinemann (2014) and estimate individual weights on the private signal for each subject according to $a_{i t}-y_{t}=c+\gamma_{i}\left(x_{i t}-y_{t}\right)+u_{i t}$, where $\gamma_{i}$ is the individual weight on the private signal. Then, we distinguish the following 12 strategy types, allowing for a slack in estimated individual weights of 0.02 :

1. agents attach about the same weight to both signals (Level 1),

2. agents weigh signals in accord with Level 2,

3. agents weigh signals in accord with Level 3,

4. agents respond to signals with equilibrium weights (level infinity),

5. agents attach weight 0 to the private signal,

6. agents attach weight 1 to the private signal,

7. agents attach weights between Level 1 and 2,

8. agents attach weights between Level 2 and 3,

9. agents attach weights between Level 3 and equilibrium weight,

10. agents put weights on the private signal between 0.5 and 1 ,

11. agents attach weights on the private signal between 0 and the equilibrium weight,

12. agents whose weights on the private signal are negative or exceed 1 ,

In a level-of-reasoning approach, strategies 5, 6, and $10-12$ are attributed to random behavior (Level 0). However, we find very different densities of these strategies. Therefore, we distinguish them. Shares of strategy types in the population are denoted by $\Phi_{1}$ to $\Phi_{12}$. To get estimates for these shares, we first look for each individual weight, whether it could be explained by either of strategies 1 to 6 allowing for a deviation of estimated weights by at most 0.02 . If an action is consistent with several of these strategies, we attribute it to the strategy that is closer. Remaining strategies can be uniquely attributed to either of the remaining types. For types 7 to 11, we assume that weights have a uniform distribution in the respective interval. For type 12, we use the actually observed weights.

For our welfare comparison, we take the distribution of strategy types estimated with data from the second half of each treatment. According to our procedure on classifying strategies, we get one distribution for each treatment laid out in Table A.

\begin{tabular}{lcccccccccccc}
\hline Strategy $s$ & 1 & 2 & 3 & 4 & 5 & 6 & 7 & 8 & 9 & 10 & 11 & 12 \\
Treatment $r=0.25$ & 40.6 & 9.4 & 0 & 6.3 & 0 & 0 & 12.5 & 0 & 0 & 21.9 & 9.4 & 0 \\
$\begin{array}{l}\text { Treatment } r=0.5 \\
\text { (standard sessions) }\end{array}$ & 26.0 & 8.3 & 6.3 & 3.1 & 1.0 & 0 & 24.0 & 0 & 0 & 17.7 & 13.5 & 0 \\
$\begin{array}{l}\text { Treatment } r=0.5 \\
\text { (control sessions) }\end{array}$ & 39.6 & 10.4 & 3.1 & 2.1 & 2.1 & 0 & 16.7 & 0 & 0 & 19.8 & 5.2 & 1.0 \\
Treatment $r=0.75$ & 21.9 & 8.3 & 2.1 & 3.1 & 12.5 & 0 & 21.9 & 3.1 & 1.0 & 13.5 & 11.5 & 1.0 \\
\hline
\end{tabular}

Table A - Shares $\Phi_{s}$ of strategy types in Treatments $r=0.5, r=0.25$, and $r=0.75$, second half, in percent. 
There were two subjects, whose estimated weights on private signals in the respective treatments were outside [0,1]: Session 20, Subject 16 has an estimated weight on the private signal of 1.04, Session 13, Subject 16 has a weight of -0.05 .

Next, we calculate algebraically the expected squared deviation of actions from the fundamental state $\theta$ for an arbitrary weight $\gamma$ on private signals

$$
E\left(\gamma x_{i}+(1-\gamma) y-\theta\right)^{2}=E\left(\gamma \varepsilon_{i}+(1-\gamma) \eta\right)^{2}=\frac{\gamma^{2}}{\beta}+\frac{(1-\gamma)^{2}}{\alpha}
$$

Note that for strategy $1, \gamma_{1}=\beta /(\alpha+\beta)$, while for level-2 players,

$$
\gamma_{2}=\frac{\alpha \beta(1-r)+\beta^{2}}{(\alpha+\beta)^{2}} \text {. }
$$

For level-3 players,

$$
\gamma_{3}=\left[(1-r)+r \gamma_{2}\right] \frac{\beta}{\alpha+\beta}=\frac{(\alpha+\beta)^{2}-r \alpha^{2}-r(1+r) \alpha \beta}{(\alpha+\beta)^{3}} \beta .
$$

For equilibrium players,

$$
\gamma^{*}=\frac{\beta(1-r)}{\alpha+\beta(1-r)} .
$$

Expected welfare loss is now given by

$$
\begin{aligned}
& \sum_{s=1}^{3} \Phi_{s}\left(\frac{\gamma_{s}^{2}}{\beta}+\frac{\left(1-\gamma_{s}\right)^{2}}{\alpha}\right)+\Phi_{4}\left(\frac{\gamma^{* 2}}{\beta}+\frac{\left(1-\gamma^{*}\right)^{2}}{\alpha}\right)+\Phi_{5} \frac{1}{\alpha}+\Phi_{6} \frac{1}{\beta}+\frac{\Phi_{7}}{\gamma_{1}-\gamma_{2}} \int_{\gamma_{2}}^{\gamma_{1}}\left(\frac{\gamma^{2}}{\beta}+\frac{(1-\gamma)^{2}}{\alpha}\right) d \gamma \\
& +\frac{\Phi_{8}}{\gamma_{2}-\gamma_{3}} \int_{\gamma_{3}}^{\gamma_{2}}\left(\frac{\gamma^{2}}{\beta}+\frac{(1-\gamma)^{2}}{\alpha}\right) d \gamma+\frac{\Phi_{9}}{\gamma_{3}-\gamma^{*}} \int_{\gamma^{*}}^{\gamma_{3}}\left(\frac{\gamma^{2}}{\beta}+\frac{(1-\gamma)^{2}}{\alpha}\right) d \gamma+\frac{\Phi_{10}}{1-\gamma_{1}} \int_{\gamma_{1}}^{1}\left(\frac{\gamma^{2}}{\beta}+\frac{(1-\gamma)^{2}}{\alpha}\right) d \gamma \\
& +\frac{\Phi_{11}}{\gamma^{*}} \int_{0}^{\gamma^{*}}\left(\frac{\gamma^{2}}{\beta}+\frac{(1-\gamma)^{2}}{\alpha}\right) d \gamma+\Phi_{12}\left(\frac{\gamma_{12}^{2}}{\beta}+\frac{\left(1-\gamma_{12}\right)^{2}}{\alpha}\right),
\end{aligned}
$$

where $\gamma_{12}$ is the actually observed weight for the subject of type 12 . Solving the integrals, the expected welfare loss equals 


$$
\begin{aligned}
& \sum_{s=1}^{3} \Phi_{s}\left(\frac{\gamma_{s}^{2}}{\beta}+\frac{\left(1-\gamma_{s}\right)^{2}}{\alpha}\right)+\Phi_{4}\left(\frac{\gamma^{* 2}}{\beta}+\frac{\left(1-\gamma^{*}\right)^{2}}{\alpha}\right)+\frac{\Phi_{5}}{\alpha}+\frac{\Phi_{6}}{\beta}+\frac{\Phi_{7}}{\gamma_{1}-\gamma_{2}}\left[\frac{\gamma_{1}^{3}-\gamma_{2}^{3}}{3 \beta}-\frac{\left(1-\gamma_{1}\right)^{3}-\left(1-\gamma_{2}\right)^{3}}{3 \alpha}\right]+ \\
& +\frac{\Phi_{8}}{\gamma_{2}-\gamma_{3}}\left[\frac{\gamma_{2}^{3}-\gamma_{3}^{3}}{3 \beta}-\frac{\left(1-\gamma_{2}\right)^{3}-\left(1-\gamma_{3}\right)^{3}}{3 \alpha}\right]+\frac{\Phi_{9}}{\gamma_{3}-\gamma^{*}}\left[\frac{\gamma_{3}^{3}-\gamma^{* 3}}{3 \beta}-\frac{\left(1-\gamma_{3}\right)^{3}-\left(1-\gamma^{*}\right)^{3}}{3 \alpha}\right]+ \\
& +\frac{\Phi_{10}}{1-\gamma_{1}}\left[\frac{1-\gamma_{1}^{3}}{3 \beta}+\frac{\left(1-\gamma_{1}\right)^{3}}{3 \alpha}\right]+\frac{\Phi_{11}}{\gamma^{*}}\left[\frac{\gamma^{* 3}}{3 \beta}+\frac{\left(1-\gamma^{*}\right)^{3}-1}{3 \alpha}\right]+\Phi_{12}\left(\frac{\gamma_{12}^{2}}{\beta}+\frac{\left(1-\gamma_{12}\right)^{2}}{\alpha}\right) \text {. } \\
& =\frac{1}{\beta}\left\{\sum_{s=1}^{3} \Phi_{s}\left(\gamma_{s}^{2}+\frac{\left(1-\gamma_{s}\right)^{2}}{\alpha / \beta}\right)+\Phi_{4}\left(\gamma^{* 2}+\frac{\left(1-\gamma^{*}\right)^{2}}{\alpha / \beta}\right)+\frac{\Phi_{5}}{\alpha / \beta}+\Phi_{6}+\frac{\Phi_{7}}{\gamma_{1}-\gamma_{2}}\left[\frac{\gamma_{1}^{3}-\gamma_{2}^{3}}{3}-\frac{\left(1-\gamma_{1}\right)^{3}-\left(1-\gamma_{2}\right)^{3}}{3 \alpha / \beta}\right]+\right. \\
& +\frac{\Phi_{8}}{\gamma_{2}-\gamma_{3}}\left[\frac{\gamma_{2}^{3}-\gamma_{3}^{3}}{3}-\frac{\left(1-\gamma_{2}\right)^{3}-\left(1-\gamma_{3}\right)^{3}}{3 \alpha / \beta}\right]+\frac{\Phi_{9}}{\gamma_{3}-\gamma^{*}}\left[\frac{\gamma_{3}^{3}-\gamma^{* 3}}{3}-\frac{\left(1-\gamma_{3}\right)^{3}-\left(1-\gamma^{*}\right)^{3}}{3 \alpha / \beta}\right]+ \\
& \left.+\frac{\Phi_{10}}{1-\gamma_{1}}\left[\frac{1-\gamma_{1}^{3}}{3}+\frac{\left(1-\gamma_{1}\right)^{3}}{3 \alpha / \beta}\right]+\frac{\Phi_{11}}{\gamma^{*}}\left[\frac{\gamma^{* 3}}{3}+\frac{\left(1-\gamma^{*}\right)^{3}-1}{3 \alpha / \beta}\right]+\Phi_{12}\left(\gamma_{12}^{2}+\frac{\left(1-\gamma_{12}\right)^{2}}{\alpha / \beta}\right)\right\} \text {. }
\end{aligned}
$$

We calculate the expected welfare loss according to this formula for several values of $r>0.5$ and $\alpha / \beta$ and check in which cases an increase in $\alpha / \beta$ raises or lowers the welfare loss. Calculations are provided in the online appendix

\section{http://www.macroeconomics.tu-berlin.de/fileadmin/fg124/heinemann/downloads/WLLR.xls}

and show that increasing the precision of public information, $\alpha$, raises expected welfare for each of the four distributions of strategies. Looking closer at these simulations, we found that this result is mainly due to the large proportion of players who put weights on the private signal that are lower than in equilibrium or higher than for Level 1 (strategy types 5, 6, and $10-12$ ). If we remove those agents and just look at remaining strategy types keeping their relative shares in the population

$$
\Phi_{s} /\left(\sum_{t=1}^{4} \Phi_{t}+\sum_{t=7}^{9} \Phi_{t}\right),
$$

we detect negative welfare effects of increasing precision of public signals for some parameter combinations with $r>0.75$ and $\beta / \alpha>20$. This result also holds for each of the four distributions and it would still hold, if the proportion of equilibrium players is much smaller. 